\title{
Spray droplet impaction models and their use within AGDISP software to predict retention
}

\author{
W.A. Forster ${ }^{1}$, G.N. Mercer ${ }^{2}$ and W.C. Schou ${ }^{3}$ \\ ${ }^{1}$ Plant Protection Chemistry NZ Ltd., PO Box 6282, Rotorua 3043, New Zealand \\ ${ }^{2}$ Australian National University, Canberra, ACT 0200, Australia \\ ${ }^{3}$ Scion, PB 3020, Rotorua 3043, New Zealand \\ Corresponding author: alison.forster@ppcnz.co.nz
}

\begin{abstract}
The retention and distribution of spray droplets within the plant canopy have a crucial effect on the biological efficacy of pesticides. To maximise spray retention, droplets that impact a leaf must remain on the plant. Three outcomes are possible when a droplet impacts a leaf surface: adhesion, bounce or shatter. Those droplets that bounce or shatter can continue their journey through the canopy, depositing at lower levels in the canopy or on the ground. Mathematical models based on the physical processes involved in the bounce/ adhesion and shatter of droplets have been developed, improved and described. These process-based retention models have recently been implemented within an experimental build of the spray application simulation software AGDISP. This has allowed differences in total spray retention to plants, due to the spray formulation used or vegetative species studied, to be predicted. This paper discusses these new tools, illustrates the effect different spray formulations and application parameters have on predicted retention, and compares model predictions with measured retention.
\end{abstract}

Keywords AGDISP, spray retention, canopy retention, bounce model, shatter model.

\section{INTRODUCTION}

The primary factors involved in the biological efficacy of pesticide sprays are: (1) deposition (as a proportion of the amount applied within the target area less drift), (2) retention (the overall capture by plants of spray droplets either on initial or subsequent impact, and after loss due to run-off), and in the case of systemic pesticides (3) uptake and (4) translocation to the site of biological activity. The level of efficacy obtained can be adversely affected by poor efficiency in any one step of the deposition-plant retentionuptake-translocation process, with in some cases as little as $1 \%$ of the spray applied reaching the site of biological activity (Zabkiewicz 2007). In order to optimise the biological efficacy of sprays, each of these factors need to be maximised, without having a detrimental effect on the other factors. As examples, deposition to the target area can be maximised by eliminating drift using very large droplets, but this leads to very low levels of spray retention, particularly on moderate through to difficult-to-wet target plants (crops or weeds); a formulation may maximise spray retention, but have a detrimental effect on uptake of a particular systemic pesticide. It must be remembered that these other factors 
are inextricably linked to determine the overall biological efficacy of sprays.

The retention and distribution of spray droplets within the plant canopy has a crucial effect on the biological efficacy of pesticides and will be the focus of this paper. To maximise spray retention, droplets that impact a leaf must remain on the plant. Three outcomes are possible on initial impact of a droplet with a leaf surface: adhesion (the droplet sticks on first impact), bounce or shatter. When an incoming droplet is intercepted by the leaf surface it first spreads out, and assuming that the droplet doesn't shatter, reaches a point of maximum spread before recoiling due to surface tension. During both the spreading and recoiling phases the droplet loses energy. If the energy loss is small enough, there is sufficient energy remaining to cause the droplet to bounce off the leaf surface. If the energy losses are larger, the droplet will adhere. A process-based (driven by physical processes and their associated physiological parameters, rather than derived from field measurements) model for bounce (Attané et al. 2007) has been improved and described (Mercer et al. 2010). This model is not applicable if the droplet shatters, and therefore there is a need to consider a droplet shatter model in parallel with the adhesion/ bounce model. For droplets with high initial kinetic energies, droplet shatter will occur if the characteristic impingement parameter $\mathrm{K}$, exceeds a critical value, $\mathrm{K}_{\text {crit }}$ (Yoon et al. 2006). If $\mathrm{K}$ is less than or equal to $\mathrm{K}_{\text {crit }}$, the droplet either bounces or adheres to the surface and the Mercer et al. (2010) model applies. A $\mathrm{K}_{\text {crit }}$ value for leaf surfaces can be estimated according to Forster et al. (2010).

AGDISP (Bilanin et al. 1989; AGDISP 8.242010 ) is a spray application simulation software package with a primary focus on aerial applications that has been developed over 30 years and is still being actively improved. A major strength of AGDISP has been its ability to model the aerial release of liquid sprays and the subsequent prediction of drift, which has been validated (Bilanin et al. 1989; Bird et al. 2002). However, AGDISP does not model spray canopy retention. Although AGDISP contained a canopy "deposition" model, this assumed that droplets that intercepted (came in contact with) a leaf surface were retained at that point (i.e. $100 \%$ adhesion). However, as previously discussed, not all droplets adhere on initial impact. Those droplets that bounce or shatter can continue their journey through the canopy, depositing at lower levels in the canopy or on the ground. Therefore, in order to predict true spray canopy retention, the process-based retention models (models for bounce and shatter) have recently been implemented (Schou et al. 2011) within an experimental build of AGDISP.

The objective of this paper is to outline the knowledge that can be gained from these shatter and bounce/adhesion models, illustrate the effect different spray formulations and application parameters have on predicted retention, and compare AGDISP and modified AGDISP predictions with measured retention.

\section{MATERIALS AND METHODS}

The materials and methods used have been described fully elsewhere. The models used to determine whether a droplet bounces or adheres are described by Mercer et al. (2010). The model used to determine shatter and the empirical equation used to calculate $\mathrm{K}_{\text {crit }}$ are described by Forster et al. (2010). Implementation of the retention models within AGDISP is described in Schou et al. (2011) and the methods used in the experimental tracksprayer spray retention studies used to compare with predicted retention results are described by Forster et al. (2006). The bounce/ adhere and shatter models both require inputs for: dynamic viscosity, density, surface tension, droplet size and velocity. In addition, the bounce model requires advancing and receding contact angle values of droplets of the formulation of interest on the species of interest, while the shatter model requires $\mathrm{K}_{\text {crit }}$, the critical value at which a droplet will shatter on the species of interest. The modified AGDISP model requires all of these inputs as well as many additional ones. The pertinent points for the inputs required by the models and software for the examples used in this study are described below. 


\section{Plant characteristics}

The wheat utilised in the tracksprayer retention trial was an average height of $26 \mathrm{~cm}$ and had a Leaf Area Index (LAI) of 3.67. This information is required by the AGDISP canopy model. LAI was estimated from plant surface areas determined using a LI-COR LI-3100 area meter and area ground cover determined using photographic images from above the plant and $\mathrm{V}^{++}$for Windows image analysis software. The average leaf angle for wheat $\left(65^{\circ}\right)$ was estimated from photographic images taken side-on of the plant. $K_{\text {crit }}$, the critical value at which a droplet will shatter, is related to plant wettability, and was estimated at 56 for wheat.

\section{Formulation characteristics: surface tension, dynamic viscosity and density}

The formulations were chosen to provide a range of surface tensions, measured using a Krüss bubble pressure tensiometer (BP2 MKII). Silwet ${ }^{\circledR}$ L-77 ${ }^{\text {TM }}$ (Momentive Performance Materials, Tarrytown, NY, USA) was used at 0.01, 0.025 and $0.1 \%$ providing static (equilibrium) surface tensions of 41,30 and $23 \mathrm{mN} / \mathrm{m}$ respectively. Water has a surface tension of $72 \mathrm{mN} / \mathrm{m}$. The dynamic viscosity and density of water $\left(0.001002 \mathrm{~kg} / \mathrm{m} / \mathrm{s}\right.$ and $998.2 \mathrm{~kg} / \mathrm{m}^{3}$, respectively) was used for all formulations. This information is required by both the bounce/adhere and the shatter models.

\section{Advancing and receding contact angle measurements}

A KSV CAM 200 optical contact angle meter with an automated tilting stage and basler digital video camera was used to determine advancing and receding contact angles of water or water plus adjuvant droplets on the wheat plant surface. Water droplets were completely repulsed by the wheat, which is a very difficult-to-wet species. It should be noted that it is practically impossible to obtain a contact angle $\geq 160^{\circ}$ as at these values the droplet will not detach from the syringe onto the plant with the droplet being completely repulsed by the plant. Therefore, the true contact angle (CA) could be any value between 160 and $180^{\circ}$.
In these instances, the advancing angle has been estimated at $179^{\circ}$ and the receding estimated at $175^{\circ}$. The advancing and receding contact angles of $0.01 \%$ and $0.025 \% \mathrm{~L}-77$ on wheat were $131^{\circ} / 107^{\circ}$ and $71^{\circ} / 46^{\circ}$ respectively. There was complete spreading of droplets containing the formulation having the lowest surface tension $(0.1 \%$ L- 77$)$, and the advancing and receding contact angles were both taken as $0^{\circ}$. Whether a droplet adheres or bounces, is also dependent on leaf angle, and average leaf angle was added to both the advancing and receding contact angles (to a maximum of $179^{\circ}$ ) to account for this. Droplets adhere less with increasing leaf angle (Forster et al. 2005), and this addition gives correct trends (W.A. Forster, personal communication), although a more robust mathematical model is still required and is the topic of current research.

\section{Bounce and shatter dropsize factors}

Assuming that the reduction in velocity caused by bouncing is proportional to the fraction of the velocity normal to the leaf surface, the bounce velocity factor was estimated using $\sin$ (average leaf angle). This gave a value of 0.91 for wheat. It has been observed (W.A. Forster, personal communication) that in general shatter reduces with increasing leaf angle, and therefore shatter was disabled (factor $=1$ ) in the case of wheat. These factors are additional inputs required to run the modified AGDISP, and are not used in either the bounce/adhere or shatter models.

\section{Other AGDISP model inputs}

The other AGDISP model inputs were assigned the following values: ground sprayer applying 150 litres/ha through 1 flat fan, ASAE fine to medium nozzle, with a boom pressure of 3 bar. The release height was $50 \mathrm{~cm}$ above plant height. Droplet velocity is calculated within AGDISP. A single height wind type, with the wind speed set as the tracksprayer belt speed (0.361, 0.45, 0.46 and $0.75 \mathrm{~m} / \mathrm{s}$ for $0.1,0.25,0.01 \% \mathrm{~L}-77$, and water, respectively), a wind direction of $-90^{\circ}$, a temperature of $20^{\circ} \mathrm{C}$ and $75 \%$ relative humidity. The atmospheric stability was overcast. The LAI canopy model with a user defined Leaf Area 
Index Envelope (plant height and cumulative LAI) was used. The element size used for wheat was $0.01 \mathrm{~m}$.

\section{RESULTS AND DISCUSSION \\ Effect of formulation, application and plant parameters on predicted shatter, adhesion or bounce}

In order to provide an interactive environment from which to run the bounce (or adhesion) and shatter models, new software tools have been developed. These allow the user to enter the variables required by the models, ie. (1) the spray formulation (surface tension, dynamic viscosity, density), (2) application (droplet size and velocity) characteristics required by both models, (3) the interaction of the spray droplet with the leaf surface (advancing and receding contact angles) required by the bounce model, and (4) the leaf characteristic (the critical value for shatter, $\mathrm{K}_{\text {crit }}$ ) required for the shatter model. The user can enter different parameters and determine the effect of changing those parameters.

For example, Figure 1a illustrates the bounce and shatter predictions for water droplets (surface tension $=72 \mathrm{mN} / \mathrm{m}$ ) ranging in size and impact velocity, impacting a very difficultto-wet plant species. The solid line is an output from the bounce model software tool; below the line the droplet is predicted to adhere, while above the line the droplet is predicted to bounce (rebound) from the surface provided it does not shatter. The dashed line, above which droplet shatter is predicted to occur, is an output from the shatter model. The effect of reducing the surface tension to $41 \mathrm{mN} / \mathrm{m}$ by altering the formulation is illustrated in Figure 1b. Larger droplets are predicted to adhere, and at higher impact velocities, than was the case for water droplets. In contrast, shatter is predicted to occur at lower droplet sizes, and lower impact velocities than was the case for the higher surface tension formulation. Reducing the surface tension still further (Figure 1c) to $23 \mathrm{mN} / \mathrm{m}$ moves the predicted bounce line still further up, beyond the axes shown on the graph. However, the shatter line again moves lower, and is now lower than the predicted bounce/adhere line, and therefore droplets below the shatter line are predicted to adhere, while those above the line will shatter. In this case, where shatter occurs first, the bounce model is not applicable and should be ignored; the droplets either adhere or shatter.

Similar results and graphs demonstrating the effects of changing the other variables required by the models (as outlined in the first paragraph of this section) can be produced, for example illustrating the effect of altering the density or dynamic viscosity of the spray formulation. All other things being equal, initial droplet adhesion is predicted to increase (and bounce decrease) with: decreasing surface tension and density of the formulation, increasing dynamic viscosity of the formulation, decreasing droplet size and droplet velocity, decreasing advancing/receding contact angles, and increasing wettability of the plant surface (results not shown). Droplet shatter is predicted to increase with: decreasing dynamic viscosity and surface tension of the formulation, increasing density of the spray formulation, increasing droplet size and droplet velocity, decreasing advancing/receding contact angles, and decreasing plant wettability.

Overall, the current models for adhesion/ bounce and shatter have shown excellent agreement with experimentally obtained values (Forster et al. 2010).

\section{Implementation of the retention models within AGDISP}

These process-based retention models for bounce (Mercer et al. 2010) and shatter (Forster et al. 2010) have recently been implemented (Schou et al. 2011) within an experimental build of the spray application simulation software AGDISP (AGDISP 8.24). To run the modified AGDISP a new user interface was developed (Schou et al. 2011) that takes a standard AGDISP.ag file with all of the inputs required for AGDISP, and adds the additional inputs needed for the retention models.

After running the modified AGDISP, most of the standard AGDISP graphs are available, along with an additional graph (e.g. Figure 2) that shows the accumulated canopy interception 
(a)

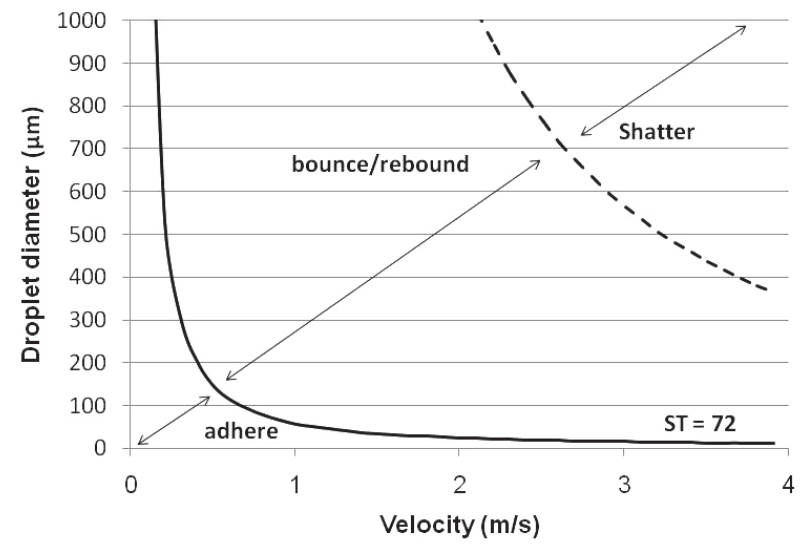

(b)

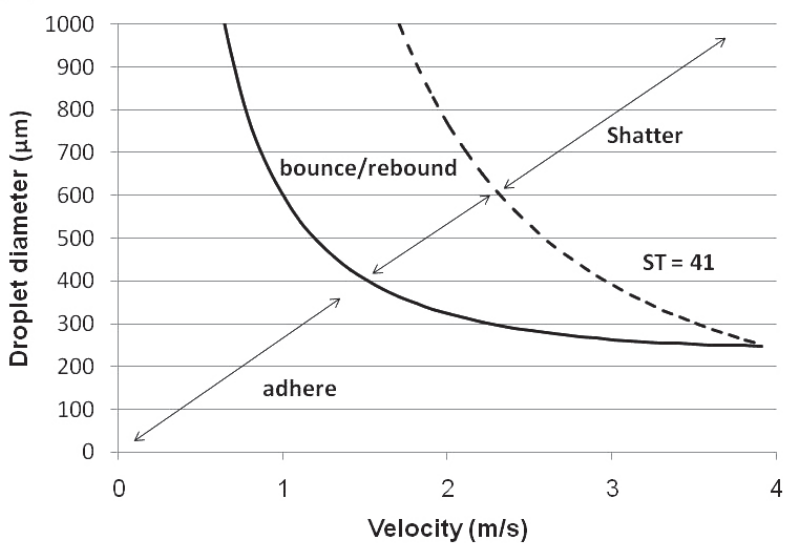

(c)

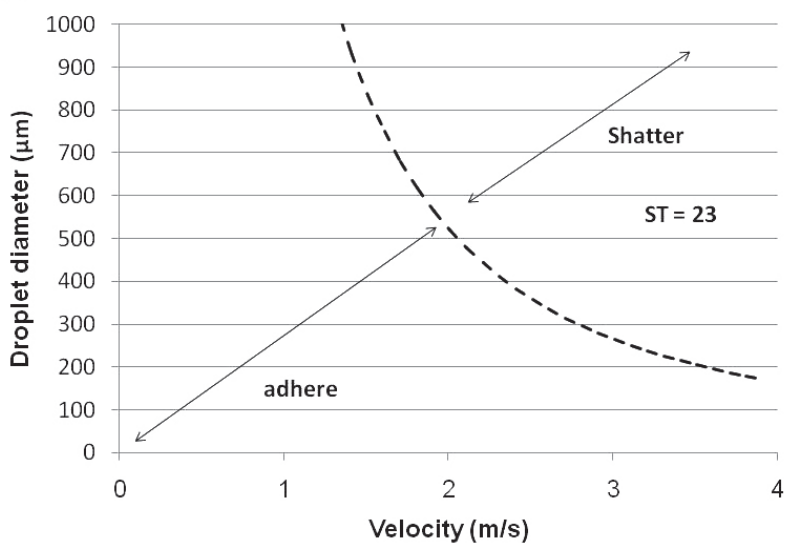

Figure 1 Droplet diameter $(\mu \mathrm{m})$ plotted against droplet velocity $(\mathrm{m} / \mathrm{s})$ to illustrate the predicted bounce (solid line) and shatter (dashed line) lines for spray droplets containing (a) water $(72 \mathrm{mN} / \mathrm{m})$, (b) a traditional adjuvant $(41 \mathrm{mN} / \mathrm{m})$ and (c) an organosilicone adjuvant $(23 \mathrm{mN} / \mathrm{m})$, impacting a very difficult-to-wet species. 
(termed deposition in the original AGDISP software) values as generated by AGDISP 8.24, together with the retention values from the modified AGDISP. The X-axis gives the vertical height of the canopy (ground $=0$ ), while the Y-axis gives the accumulated volume fraction (either intercepted, retained or adhesion on initial impact). The adhesion curve shows the number of drops that adhere on first interception, and is only useful in aiding the understanding of the processes occurring. The difference between the adhesion line and the retained line is due to droplets either bouncing or shattering, and then being retained further down in the canopy. The number of droplets adhering on initial impact is simply a subset of the total retained. It is interesting to note, however, that if the original AGDISP assumption was correct, i.e. that all droplets that intercept a leaf surface are retained at that point, then the adhesion line would equal the intercepted line. In the case illustrated in Figure 2, of the spray intercepted, $49 \%$ is predicted to be retained and therefore $51 \%$ is predicted to land on the ground beneath the plant. It can be seen (Figure 2) that the retention model provides a profile of canopy retention that can look quite different than the original AGDISP deposition line that has been more correctly termed interception in the current paper. The ability to accurately model retention through the canopy in this way will enable specific areas in the canopy to be targeted.

\section{Comparison of model predictions with measured retention}

Model predictions for wheat (Schou et al. 2011) were compared to previously determined laboratory-based tracksprayer retention to wheat (Forster et al. 2006). Figure 3 presents the results for total canopy interception and retention to wheat, sprayed with four formulations varying in surface tension $(23-72 \mathrm{mN} / \mathrm{m})$, as a percent of volume applied. There was no difference in predicted interception to wheat between the formulations, while predicted retention using the modified (to include bounce and shatter) AGDISP was much lower and did show differences between the formulations used. It can be seen

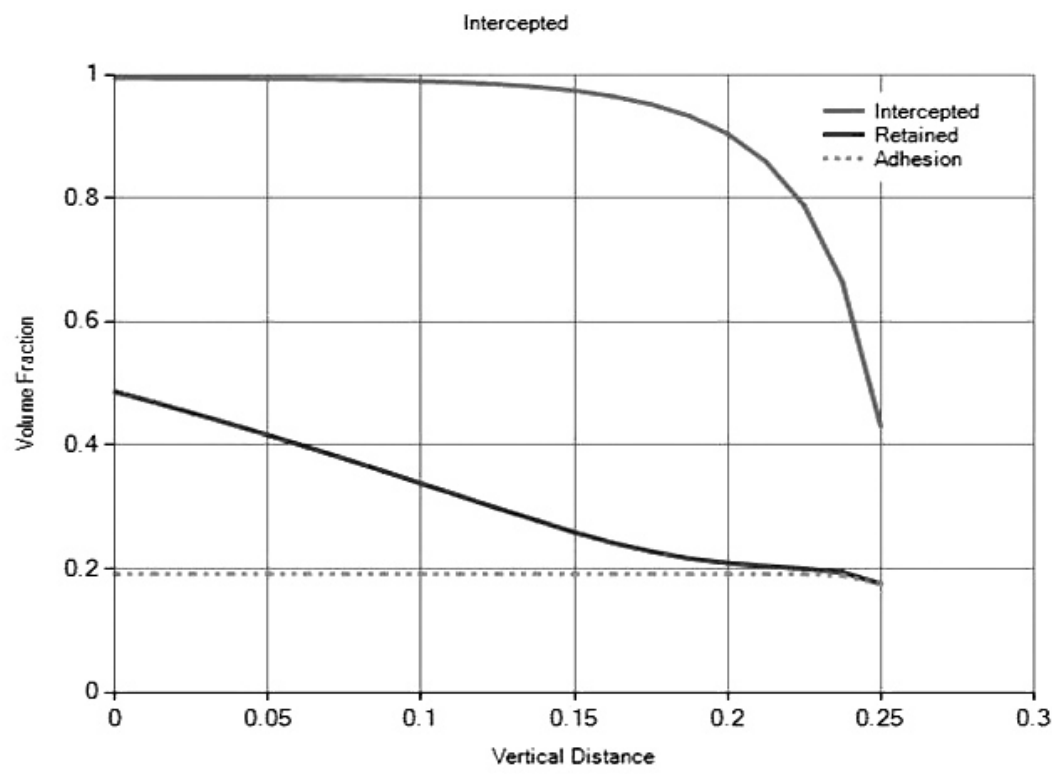

Figure 2 Predicted adhesion, retention and interception of $0.025 \%$ Silwet ${ }^{\circledR} \mathrm{L}-77^{\mathrm{TM}}$ to wheat. The X-axis gives the vertical height of the canopy ( $\mathrm{m}$; ground $=0$ ), while the $\mathrm{Y}$-axis gives the accumulated volume fraction (either intercepted, retained or adhesion on initial impact). 
that predicted retention to wheat is much more comparable to the experimentally determined retention to wheat (from Forster et al. 2006) than predicted interception, for all formulations studied. This is to be expected now that it is understood that the original AGDISP software modelled canopy interception (misleadingly termed deposition in most literature) of the spray, and not what occurs after spray interception. The only effect that formulation could have had on spray interception within the original AGDISP model was any differing effect on the spray droplet spectrum produced or evaporation rate of the droplets. These differences were considered minor in the current example. However, small differences in formulation properties can lead to large differences in the proportion of spray droplets that adhere, bounce or shatter after droplet interception, and therefore large differences in spray retention to the plant.

\section{CONCLUSIONS}

Prototype interactive software tools have been developed to run models of bounce (or adhesion) and shatter for spray droplets impacting leaf surfaces. These allow the user to enter the variables required by the models. The user can enter different parameters and determine the effect of changing those parameters on spray droplet adhesion/bounce or shatter.
The original AGDISP canopy interception model was unable to fully account for differences in retention due to the spray formulation used. The incorporation of the process-driven models for bounce and shatter has allowed these differences to be predicted, with excellent agreement in relation to laboratory-based tracksprayer retention results. Considerable work is still required to make this approach practical.

These developments should make it possible to tailor spray formulations, as well as application technologies, to maximise retention to plant foliage while minimising loss to the ground, and target specific areas in the canopy if required. These models provide a key component for the optimisation of the biological efficacy of sprays. Experiments are necessary to evaluate, calibrate, and characterise uncertainty. Once completed, this software can be used to investigate effects of variations in formulation without the need for large-scale, time-consuming and costly experiments.

\section{ACKNOWLEDGEMENTS}

This research was funded by the New Zealand Ministry of Science and Innovation (MSI; Contract No. LVLX0901: Protecting NZ's Environment; adhere/bounce or shatter models were developed within Contract No. C10X0811: Undermining Weeds).

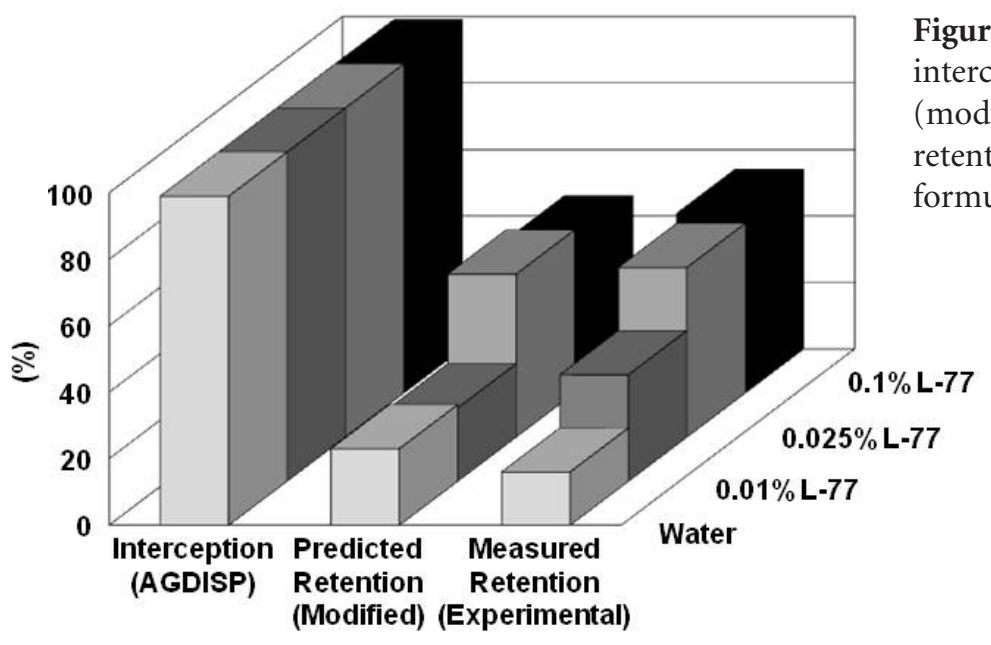

Figure 3 Predicted percent spray interception (AGDISP) and retention (modified AGDISP), and measured retention, using four different spray formulations on wheat. 


\section{REFERENCES}

AGDISP 8.24 2010. USDA Forest Service Spray Modeling Software, version 8.24, 2010. Harold W. Thistle, USDA Forest Service, 180 Cranford Street, Morgantown, WV, 26505, USA.

Attané P, Girard F, Morin V 2007. An energy balance approach of the dynamics of drop impact on a solid surface. Physics of Fluids 19: $1-17$.

Bilanin AJ, Teske ME, Barry JW, Ekblad RB 1989. AGDISP: The aircraft spray dispersion model, code development and experimental validation. Transactions of the ASAE 32 (1): 327-334.

Bird SL, Perry SG, Ray SL, Teske ME 2002. Evaluation of the AGDISP aerial spray algorithms in the AgDRIFT model. Enviromental Toxicology and Chemistry 21 (3): 672-682.

Forster WA, Mercer GN, Schou WC 2010. Processdriven models for spray droplet shatter, adhesion or bounce. Proceedings of the $9^{\text {th }}$ International Symposium on Adjuvants for Agrochemicals: 277-285.

Forster WA, Kimberley MO, Steele KD, Haslett MR, Zabkiewicz JA 2006. Spray retention models for arable crops. Journal of ASTM International 3(6): Paper ID JAI13528. www. astm.org (accessed 15 April 2012).
Forster WA, Zabkiewicz JA, Kimberley MO 2005. A universal spray droplet adhesion model. Transactions of the ASAE 48(4):1321-1330.

Mercer GN, Sweatman WL, Forster WA 2010. A model for spray droplet adhesion, bounce or shatter at a crop leaf surface. In: Fitt AD, Norbury J, Ockendon H, Wilson E ed. Progress in Industrial Mathematics at EMCI 2008. Mathematics in Industry 15, DOI: 10.1007/978-3-642-12110-4_151. SpringerVerlag, Berlin, Heidelberg. Pp. 937-943.

Schou WC, Forster WA, Mercer GN, Teske ME, Thistle HW 2011. Building canopy retention into AGDISP: Preliminary models and results. ASABE Meeting, Louisville, Kentucky. Paper No. 1110717.

Yoon SS, DesJardin PE, Presser C, Hewson JC, Avedisian CT 2006. Numerical modelling and experimental measurements of water spray impact and transport over a cylinder. International Journal of Multiphase Flow 32: 132-157.

Zabkiewicz JA 2007. Spray formulation efficacy - holistic and futuristic perspectives. Crop Protection 26: 312-319. 